\title{
Vasospasm causing a cold hemiplegic arm
}

\author{
P Wanklyn, JM Bamford
}

\begin{abstract}
Summary
We report a case of a patient with abnormal coldness of the hemiplegic arm following a stroke.
\end{abstract}

Keywords: hemiplegic arm, vasospasm

Several phenomena may occur exclusively in the hemiplegic arm. Examples are an increased sensitivity to ultraviolet light resulting in erythema ${ }^{1}$ and finger clubbing. ${ }^{2}$ Other phenomena may show a relative sparing of the hemiplegic arm. Patients who have generalised arthritis ${ }^{3}$ or endogenous eczema ${ }^{4}$ have been reported to have less severe disease of the paralysed extremity (see box 1). An abnormal sensation of coldness in the hemiplegic arm is reported to occur in more than $50 \%$ of patients with hemiplegic stroke. ${ }^{5}$ Most cases are mild but in around $12 \%$ the symptom is constant and troublesome. This symptom may not be familiar to many clinicians and this may be because only about a third of patients with the symptom complain about it to a doctor. ${ }^{5}$

\section{Case report}

A right-handed, 37-year-old woman, suffered a stroke in 1992. Right-sided weakness involving the face, arm and leg was accompanied by mild headache, dysarthria and mild spinothalamic sensory loss but no dysphasia or visual field defect. The clinical diagnosis was of the lacunar syndrome of sensorimotor stroke and a computed tomography (CT) scan showed a small infarct in the posterior limb of the internal capsule compatible with occlusion of a single perforating artery. No source of cardiac or artery-to-artery embolism was found on full investigation. She was a nonsmoker and was not hypertensive or diabetic. Her medications were progesterone and zopiclone. She was

\footnotetext{
Medical Department for the Elderly, Leeds General Infirmary, Leeds LS1 3EX, UK P Wanklyn

\section{Department of Neurology, St James' University Hospital, Leeds, UK JM Bamford}

Phenomena which may occur in the hemiplegic arm

- unpleasant coldness

- sparing of eczema

- sparing of arthritis

- increased sensitivity to ultra-violet light

- pitting oedema

- shoulder pain and subluxation

- reflex sympathetic dystrophy

- unilateral clubbing

Box 1 given low-dose aspirin for secondary stroke prevention and was eventually discharged home.

Since the age of 30 years she had noted that her hands became symmetrically cold and changed colour from pink to white and back to red even in mildly cold weather. She had not noticed any relationship of these symptoms to her menstrual cycle and had not used vibrating tools at work.

She was referred 18 months after the stroke because of unpleasant coldness in the hemiplegic arm. This coldness was similar to the feeling she had previously noticed in both hands but was much more severe on the hemiplegic side. Rubbing the arm and exercise improved the coldness but the benefit was limited. She had no current arm swelling, pain, or change in skin colour but had noticed increased sweating of the affected arm. She had a mild spinothalamic sensory loss and moderately severe weakness but no evidence of any new neurological event and no metacarpophalangeal joint tenderness. Her pulses were equal and blood pressure was $120 / 80 \mathrm{mmHg}$ on the left and $124 / 80 \mathrm{mmHg}$ on the right.

Further evaluation was undertaken using a laser doppler flowmeter (Moor Instruments, Axminster, Devon). The doppler flowmeter measures 'red cell flux' which is the product of red cell concentration and velocity. This measure of flux is an estimate of cutaneous blood flow. ${ }^{6}$ Readings were taken at the patient's home in a quiet room with the patient seated and relaxed. Two laser probes were used to measure blood flux simultaneously from the index finger of each hand with readings taken at a rate of 5/s with the arms at the level of the heart. Once the trace was steady, a prolonged reading of flux was obtained and repeated three times and the mean flux calculated. Two sets of readings were taken on different days (see table). It can be seen that finger skin flow was reduced on the hemiplegic side on both days. It is also interesting that the assymmetry in flow was accentuated on the warmer day, probably as a result of abnormal unilateral vasoconstriction in the cutaneous vessels on the hemiplegic side.

Table 1 Blood flux in the two hands measured simultaneously

\begin{tabular}{lll}
\hline & \multicolumn{2}{l}{ Mean finger blood flux (flux units) } \\
\cline { 2 - 3 } & Hemiplegic arm & Unaffected arm \\
\hline Day 1 $\left(19^{\circ} \mathrm{C}\right)$ & 20.8 & 35.1 \\
Day 2 $\left(25^{\circ} \mathrm{C}\right)$ & 42 & 124 \\
\hline
\end{tabular}




\section{Discussion}

There are a number of potential causes of this abnormal cold sensation (see box 2). Coldness in the limbs may occur in patients with reflex sympathetic dystrophy after stroke.

Reflex sympathetic dystrophy has a combination of typical features in the affected limb including pain, swelling, vasomotor instability and functional limitation. It has been variably defined in the past and there are no true diagnostic tests (triple phase bone scan has proved useful and may be more sensitive than clinical examination). ${ }^{7}$ One recent report included a comprehensive definition which may be helpful (see box 3$)^{8}{ }^{8}$ The condition may be more difficult to diagnose in stroke patients for several reasons. Pain may occur in the hemiplegic arm for many other reasons such as shoulder capsulitis, muscular pain, or central ('thalamic') pain. Oedema due to dependency and disuse is common and limited range of movement is usually due to muscular weakness. Estimates of the prevalence of reflex sympathetic dystrophy in hemiplegia vary from $12.5 \%{ }^{9}$ to $25 \% .{ }^{7}$ One clinical feature which was found to be predictive of the future development of reflex sympathetic dystrophy was tenderness over the metacarpopharyngeal joints of the affected hand. ${ }^{7}$ Sensory deficits might cause abnormal sensations in the limbs but if this were a common cause of the coldness one would expect a large proportion of patients with a left hemiplegia to suffer and this has not been observed in studies to date.

Another possible cause of coldness in the hemiplegic arm is a primary vasomotor abnormality. We have also shown that stroke patients with abnormal coldness in the hemiplegic arm have reduced blood flow to the affected hand and slower rewarming of the arm after cold stress. ${ }^{10} \mathrm{~A}$ group of stroke patients with no coldness in the arm had no significant vasomotor abnormalities.

The patient described here had symptoms suggestive of Raynaud's phenomenon beginning seven years before the stroke and affecting both hands equally. After the stroke, the hemiplegic arm felt much colder to the patient. There were no other features suggestive of reflex sympathetic dystrophy except excessive

1 Cox N, Williams S. Lowered ultraviolet minimal erythema dose in hemiplegia. Postgrad Med $\mathcal{F}$ 1985; 61: 575-7.

2 Kahtan S, Kahtan N. Unilateral finger clubbing. Lance 1991; 338: 576.

3 Mitchell W, Capell H. Unexplained arthritis in hemiplegia. BMf 1982; 284: 86 .

4 Troilius A, Moller H. Unilateral eruption of endogenous eczema after hemiparesis. Acta Dermatol Venereol (Stockh) 1989; 69: 256-8.

5 Wanklyn P, Mulley G. The cold hemiplegic arm: a community survey. Age Ageing 1994; 23 (suppl 4): 19 (abstract). 6 Oberg P, Tenland T, Nilssen G. Laser doppler flowmetry: a non invasive and continuous method for blood flow evaluation in microvascular studies. Acta Med Scand 1983; 687 (suppl): 17-24.
Possible causes of a unilateral coldness in the hemiplegic arm

- reflex sympathetic dystrophy

- vasomotor disturbance due to the stroke

- sensory deficits

- exacerbation of underlying vasospastic disorder by stroke

Box 2

Reflex sympathetic dystrophy: definition $^{8}$

Four or five of the following:

- unexplained diffuse pain

- difference in skin colour

- diffuse oedema

- difference in skin temperature

- limited range of movement in affected limb

Symptoms are worse after use and occur

diffusely in the limb

Box 3

\section{Learning points}

- coldness in the hemiplegic arm may be a distressing symptom after stroke

- causes include excessive vasoconstriction, reflex sympathetic dystrophy and possibly sensory dysfunction

- further research into potential treatments is needed

Box 4

sweating. The marked worsening of the symptom of coldness and reduced flux on the hemiplegic side implies this patient probably has a vasospastic disorder which has been exacerbated by the stroke.

There have been no trials investigating treatment specifically for the cold hemiplegic arm. Patients tend to use simple local warming and wrapping of the arm. ${ }^{5}$ Reflex sympathetic dystrophy has been treated with sympathectomy, guanethidine blocks, and calcium antagonists ${ }^{8}$ but the relief of pain and improvement in function have been the usual objectives.

7 Tepperman P, Greyson D, Hilbert L, Jimenez J, Williams J. Reflex sympathetic dystrophy in hemiplegia. Arch Phys Med Rehabil 1984; 65: 442-6.

8 Veldman P, Reynan H, Arntz I, Goris R. Signs and symptoms of reflex sympathetic dystrophy: prospective
study of 829 patients. Lancet 1993; 342: 1012-6.

9 Davis S, Petrillo C, Eichberg R, Chu D. Shoulder hand syndrome in a hemiplegic population: 5 -year retrospective syndrome in a hemiplegic population: 5-year ret
study. Arch Phys Med Rehabil 1977; 58: 353-6.

10 Wanklyn P, Ilsley D, Roper TA, Greenstein D, Kester R, Mulley GP. The cold hemiplegic arm. Stroke 1994; 25: 1765-70. 